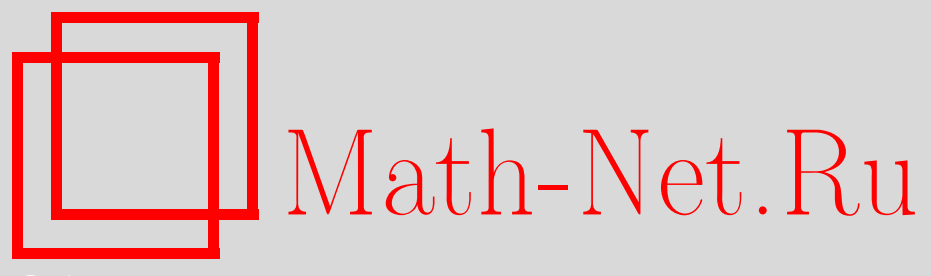

В. М. Бухштабер, Д. В. Лейкин, Дифференцирование абелевых функций по параметрам, УМH, 2007, том 62, выпуск 4, 153-154

DOI: https://doi.org/10.4213/rm7598

Использование Общероссийского математического портала Math-Net.Ru подразумевает, что вы прочитали и согласны с пользовательским соглашением http://www.mathnet.ru/rus/agreement

Параметры загрузки:

IP: 54.80 .97 .219

26 апреля 2023 г., 16:09:35

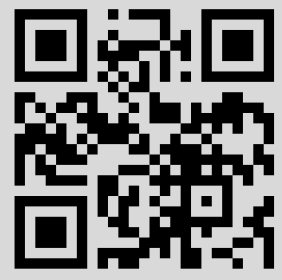




\section{Дифференцирование абелевых функций по параметрам}

\section{В. М. Бухштабер, Д. В. Лейкин}

Отождествим $\mathbb{C} \cup \infty$ с $S^{2}$ и рассмотрим $f(x, y, \lambda)$ - полином по $x, y$ и $\lambda$, где $x$ и $y$ из $S^{2}$ и $\lambda=\left(\lambda_{1}, \ldots, \lambda_{d}\right) \in \mathbb{C}^{d}$. Мы будем предполагать, что $f(x, y, \lambda)=0$ задает $y(x, \lambda)$ как целую алгебраическую функцию $x$ и голоморфную функцию $\lambda$.

Рассмотрим семейство кривых $V=\left\{(x, y, \lambda) \in\left(S^{2}\right)^{2} \times \mathbb{C}^{d} \mid f(x, y, \lambda)=0\right\}$ и проекцию $p: V \rightarrow \mathbb{C}^{d}$. Зафиксируем целое число $g>0$ и обозначим через $B=B(g)$ подмножество всех точек $b \in \mathbb{C}^{d}$ таких, что род кривой $V_{b}=p^{-1}(b)$ равен $g$. Пусть $A(x, y, \lambda)^{t}=\left(a_{k}(x, y, \lambda) \mathrm{d} x\right), k=1, \ldots, g,-$ вектор 1-форм на $V$, голоморфных по $\lambda$, такой, что $A(x, y, b)$ - вектор базисных дифференциалов первого рода на $V_{b}$ для всех $b \in B$. Пусть $V_{\circ}$ - семейство кривых $V_{\circ b}=V_{b} \backslash(\infty)$ с выколотой точкой в бесконечности. Пусть $A^{*}(x, y, \lambda)^{t}=\left(a_{k}^{*}(x, y, \lambda) \mathrm{d} x\right), k=1, \ldots, g,-$ вектор 1-форм на $V_{\circ}$, голоморфных по $\lambda$, такой, что $D(x, y, b)^{t}=\left(A(x, y, b)^{t}, A^{*}(x, y, b)^{t}\right)$ - вектор базисных голоморфных дифференциалов на $V_{\circ}$ для всех $b \in B$.

Лемма 1. Для произвольного векторного поля $\ell=\sum_{i=1}^{d} \ell_{i}(\lambda) \partial_{\lambda_{i}}$, где $\ell_{i}(\lambda)-м$ мероморфные функиии, существует единственная матрица $\Gamma_{\ell}(\lambda)$ такая, что дифференциал $\ell(D(x, y, b))+\Gamma_{\ell}(b) D(x, y, b)$ является точным на $V_{\circ ь}$ для всех $b \in B$.

Далее мы будем предполагать, что вектор $D(x, y, \lambda)$ выбран так, что его матрица периодов $\Omega(\lambda)$ удовлетворяет соотношению Лежандра $(\Omega(b))^{t} J \Omega(b)=2 \pi \sqrt{-1} J$ для всех $b \in B$, где $J=\left(\begin{array}{cc}0_{g} & 1_{g} \\ -1_{g} & 0_{g}\end{array}\right)$. Тогда матрица $M_{\ell}(\lambda)=J \Gamma_{\ell}(\lambda)=\left(\begin{array}{cc}\gamma_{\ell} & \beta_{\ell} \\ \delta_{\ell} & \alpha_{\ell}\end{array}\right)$ - симметрическая, т. е. $\alpha_{\ell}=\alpha_{\ell}^{t}, \gamma_{\ell}=\gamma_{\ell}^{t}$ и $\delta_{\ell}=\beta_{\ell}^{t}$, где $\alpha_{\ell}=\left(\alpha_{k l}\right), \beta_{\ell}=\left(\beta_{l}^{k}\right)$ и $\gamma_{\ell}=\left(\gamma^{k l}\right)-$ $(g \times g)$-матрицы. Пусть $u=\left(u_{1}, \ldots, u_{g}\right) \in \mathbb{C}^{g}$. Положим

$$
H_{\ell}=\alpha_{k l} \partial_{u_{k}, u_{l}}+2 \beta_{l}^{k} u_{k} \partial_{u_{l}}+\gamma^{k l} u_{k} u_{l} .
$$

Здесь и ниже подразумевается суммирование от 1 до $g$ по повторяющимся индексам.

В работе [1] построена сигма-функция $\sigma(u, \lambda)$ - единственная голоморфная функция, которая является совместной собственной функцией операторов ковариантного сдвига $S_{j}=\exp \left\{\eta_{i}^{j}(\lambda) u_{i}\right\} \exp \left\{\omega_{i, j}(\lambda) \partial_{u_{i}}\right\}$, где $\left(\left(\omega_{i, j}(\lambda)\right)^{t},\left(\eta_{i}^{j}(\lambda)\right)^{t}\right)^{t}, i=1, \ldots, g,-$ $j$-й столбец матрицы $\Omega(\lambda), j=1, \ldots, 2 g$, и оператора $2 \ell-H_{\ell}$ для любого мероморфного векторного поля $\ell$. Положим $\zeta_{k}(u, \lambda)=\partial_{u_{k}} \log \sigma(u, \lambda), k=1, \ldots, g$.

Обозначим через $\mathscr{F}$ поле мероморфных функций $F(u, \lambda)$ таких, что для каждого $b \in B$ функции $F(u, b)$ образуют поле $\mathscr{F}_{b}$ абелевых функций с периодами, порожденными $\left(\omega_{1, i}(b), \ldots, \omega_{g, i}(b)\right), i=1, \ldots, 2 g$.

Теорема 2. Для произвольного мероморфного векторного поля $\ell=\sum_{i=1}^{d} \ell_{i}(\lambda) \partial_{\lambda_{i}}$ оператор $d_{\ell}=\ell-\alpha_{k l} \zeta_{k}(u, \lambda) \partial_{u_{l}}-\beta_{l}^{k} u_{k} \partial_{u_{l}}$ является дифференцированием поля $\mathscr{F}$.

ДокАзАтЕльство. Используя классические результаты теории абелевых функций, можно показать, что если теорема верна хотя бы для одной непостоянной по $u$ функции из $\mathscr{F}$, то она верна для всех функций из $\mathscr{F}$. Далее, сигма-функция удовлетворяет уравнению $\left\{2 \ell-H_{\ell}\right\} \sigma(u, \lambda)=E_{\ell}(\lambda) \sigma(u, \lambda)$, где $E_{\ell}(\lambda)$ - мероморфная функция, которая для данного семейства кривых $V$ полностью определяется векторным полем $\ell$. Имеем: $\wp_{i j}(u, \lambda)=-\partial_{u_{i}, u_{j}} \log \sigma(u, \lambda) \in \mathscr{F}$ и $\partial_{u_{i}, u_{j}}\left(\sigma^{-1}\left\{2 \ell-H_{\ell}-E_{\ell}\right\}(\sigma)\right)=$ $-2 d_{\ell}\left(\wp_{i j}\right)+\alpha_{k l}\left(\partial_{u_{k}, u_{l}} \wp_{i j}-2 \wp_{i k} \wp_{j l}\right)+2 \beta_{k}^{j} \wp_{i k}+2 \beta_{k}^{i} \wp_{j k}-2 \gamma_{i j}=0 \Rightarrow d_{\ell}\left(\wp_{i j}\right) \in \mathscr{F}$.

ЗАмечание 3. Формулировка теоремы в терминах связности в расслоении, ассоциированном с расслоением $p: V \rightarrow B$, дана в [2]. Дифференцирования поля эллиптических функций были впервые найдены в [3].

Рассмотрим кольцо $\mathscr{R} \subset \mathscr{F}$ функций, голоморфных по $\lambda$. В качестве образующих кольца $\mathscr{R}$ можно выбрать $\lambda_{1}, \ldots, \lambda_{d}$ и $\wp_{i_{1} \cdots i_{k}}(u, \lambda)=-\partial^{k} \log \sigma(u, \lambda) / \partial u_{i_{1}} \cdots \partial u_{i_{k}}$, $k>1$. 
Пусть теперь $B=\mathbb{C}^{d} \backslash \Sigma$, где $\Sigma$ - гиперповерхность, заданная неприводимым алгебраическим уравнением $\Delta(\lambda)=0$. Пусть $\ell$ - голоморфное векторное поле, касательное к $\Sigma$, т. е. такое, что $\ell \log \Delta(\lambda)$ - голоморфная функция. Из общих результатов теории особенностей [4] следует, что для такого поля $\ell$ матрица $\Gamma_{\ell}(\lambda)$ голоморфна.

СледСтвиЕ 4. Для поля $\ell=\sum_{i=1}^{d} \ell_{i}(\lambda) \partial_{\lambda_{i}}$, оператор $d_{\ell}$ является дифференцированием кольца $\mathscr{R}$.

В приложениях важную роль играют семейства $V$, заданные квазиоднородными полиномами $f(x, y, \lambda)$, когда существуют ненулевые наборы целых чисел $\varepsilon_{f}, \varepsilon_{x}, \varepsilon_{y}$ и $\varepsilon_{1}, \ldots, \varepsilon_{d}$ такие, что $\varepsilon_{x} x \partial_{x} f+\varepsilon_{y} y \partial_{y} f+\sum_{k=1}^{d} \varepsilon_{k} \lambda_{k} \partial_{\lambda_{k}} f=\varepsilon_{f} f$. В этом случае при $\operatorname{deg} u_{i}=\varepsilon_{x}+\operatorname{deg} a_{i}(x, y, \lambda), i=1, \ldots, g$, сигма-функция становится квазиоднородной.

СледСтвие 5. Пусть полином $f(x, y, \lambda)$ квазиоднороден $u \varepsilon_{k}>0, k=1, \ldots, d$. Тогда если $\ell=\sum_{i=1}^{d} \ell_{i}(\lambda) \partial_{\lambda_{i}}$ - голоморфное квазиоднородное векторное поле, касающееся $\Sigma, u \operatorname{deg} \ell>0$, то матрища $\Gamma_{\ell}(\lambda)$ полиномиальна.

Пространство $H(\Sigma)$ квазиоднородных голоморфных векторных полей, касающихся $\Sigma$, является градуированным модулем над градуированным кольцом квазиоднородных голоморфных функций от $\lambda$.

СледСтвиЕ 6. Пусть $\varepsilon_{k}>0, k=1, \ldots, d, u$ в модуле $H(\Sigma)$ существует базис $L_{1}, \ldots, L_{d+q}, q \geqslant 0$, такой, что $\operatorname{deg} L_{i} \geqslant 0, k=1, \ldots, d+q$. Тогда коэфбициенты разложения $\sigma(u, \lambda)$ в ряд по степеням $u_{1}, \ldots, u_{g}$ являются полиномами от $\lambda$.

Этот результат применим к семействам $(n, s)$-кривых (см. [1], [2]), в которые включаются модели всех неприводимых алгебраических кривых.

ПримеР: СЕмЕйство кривых $f(x, y, \lambda)=y^{2}-4 x^{2 g+1}-\sum_{k=0}^{2 g-1} \lambda_{k} x^{k}$. Имеем $d=2 g$, $\varepsilon_{x}=2, \varepsilon_{y}=2 g+1, \varepsilon_{k}=2(2 g+1-k), k=0, \ldots, 2 g-1$. Производящая функция базиса в $H(\Sigma)$ задается (см. [5]) соотношением $x^{2 g-1} \sum_{k=0}^{2 g-1} x^{-k} L_{2 k} \equiv \sum_{k=0}^{2 g-1} f \mathrm{~d}_{x}^{k+1}\left(\partial_{x} f\right) \partial_{\lambda_{k}}$, где 三 обозначает сравнение по модулю идеала $\left(\partial_{x} f, \partial_{y} f\right)$ и $\mathrm{d}_{x}-$ линейный оператор на $\mathbb{C}[x]$ такой, что $\mathrm{d}_{x} 1=0$ и $\mathrm{d}_{x} x^{j}=x^{j-1}, j>0$. Ясно, что $\operatorname{deg} L_{2 k}=2 k$. Возьмем $a_{i}(x, y, \lambda)=x^{i-1} / y$, тогда $\operatorname{deg} u_{i}=-2(g-i)-1, i=1, \ldots, g$.

Теорема 7. Базис модуля дифференцирований гиперэллиптического кольца Я дается операторами $\partial_{u_{1}}, \ldots, \partial_{u_{g}} u d_{L_{2 k}}, k=0, \ldots, 2 g-1$. Производящая функиия операторов $d_{L_{2 k}}$ задается соотношением $x^{2 g-1} \sum_{k=0}^{2 g-1} x^{-k} d_{L_{2 k}} \equiv \sum_{k=0}^{2 g-1} f \mathrm{~d}_{x}^{k+1}\left(\partial_{x} f\right) \partial_{\lambda_{k}}+$ $\sum_{i=1}^{g} \sum_{j=1}^{i-2} x^{i-j-2} j u_{j} f \partial_{u_{i}}-\sum_{i, j=1}^{g}\left(2 x^{i+j-2} \zeta_{j}+\frac{1}{2} x^{i+j-1} u_{j} \partial_{x} \mathrm{~d}_{x}^{2 i}(f)\right) \partial_{u_{i}}$.

\section{Список литературы}

[1] В. М. Бухштабер, Д. В. Лейкин, Функи. анализ и его прилож., 38:2 (2004), 12-27. [2] V. M. Buchstaber, D. V. Leykin, The problem of differentiation of an Abelian function over its parameters, MIMS Preprint 2006.414. [3] F. G. Frobenius, L. Stickelberger, J. Reine Angew. Math., 92 (1882), 311-337. [4] В. И. Арнольд, А. Н. Варченко, С. М. Гусейн-Заде, Особенности дифференцируемых отображений, МЦНМО, М., 2004. [5] В. М. Бухштабер, Д. В. Лейкин, Функи. анализ и его прилож., 36:4 (2002), 18-34.

\section{B. М. Бухштабер (V. M. Buchstaber)}

Математический институт им. В. А. Стеклова РАН

E-mail: buchstab@mi.ras.ru

Д. В. Лейкин (D. V. Leikin)

Институт магнетизма, Киев, Украина

E-mail: dile@imag.kiev.ua
Представлено С. П. Новиковым Принято редколлегией 01.06.2007 